\title{
Statistical survey of day-side magnetospheric current flow using Cluster observations: magnetopause
}

\author{
Evelyn Liebert, Christian Nabert, Christopher Perschke, Karl-Heinz Fornaçon, and Karl-Heinz Glassmeier \\ Institut für Geophysik und extraterrestrische Physik, Technische Universtität Braunschweig, Braunschweig, Germany \\ Correspondence to: Evelyn Liebert (e.liebert@tu-bs.de)
}

Received: 2 December 2016 - Revised: 21 April 2017 - Accepted: 21 April 2017 - Published: 17 May 2017

\begin{abstract}
We present a statistical survey of current structures observed by the Cluster spacecraft at high-latitude day-side magnetopause encounters in the close vicinity of the polar cusps. Making use of the curlometer technique and the fluxgate magnetometer data, we calculate the 3-D current densities and investigate the magnetopause current direction, location, and magnitude during varying solar wind conditions. We find that the orientation of the day-side current structures is in accordance with existing magnetopause current models. Based on the ambient plasma properties, we distinguish five different transition regions at the magnetopause surface and observe distinctive current properties for each region. Additionally, we find that the location of currents varies with respect to the onset of the changes in the plasma environment during magnetopause crossings.
\end{abstract}

Keywords. Magnetospheric physics (current systems; magnetopause cusp and boundary layers; instruments and techniques)

\section{Introduction}

In simple magnetospheric current models the low-latitude current on the day-side magnetopause flows from dawn to dusk. At higher latitudes it is partially closed via dusk-dawndirected currents lobe-ward of the cusp (Fig. 1). This picture was also confirmed by magnetohydrodynamic (MHD) simulations for nominal solar wind conditions. If the solar wind becomes a low Mach number flow, the current distributions derived from the simulations are altered and the direction of the high-latitude portion of the magnetopause current changes its direction into a dawn-dusk orientation (e.g. Lopez et al., 2011).
Previous space missions allowed in situ investigations of several magnetopause properties based on single or dualspacecraft measurements. In such cases, it is possible to estimate a current density calculating the ratio of the jump in the magnetic field and a derived thickness of the current layer. Since 2000 the multi-spacecraft mission Cluster (Escoubet et al., 2001) has enabled simultaneous magnetic field measurements at the vertices of a tetrahedron formed by its four spacecraft. Applying the so-called curlometer technique (Dunlop et al., 1988), the Cluster mission enabled a new approach for the in situ 3-D current density determination.

The curlometer has been used in several studies, but when applied to the day-side magnetopause often only a few selected events have been investigated as case studies (e.g. Dunlop et al., 2001, 2002; Dunlop and Balogh, 2005; Panov et al., 2006). Panov et al. (2008) performed a statistical study of different properties of 154 magnetopause crossings including the current density magnitude calculated via the curlometer technique, but they did not analyse the calculated current directions. Using magnetic field data from about 4000 Cluster magnetopause crossing events, Anekallu et al. (2013) were able to perform a larger statistical study based on single-spacecraft measurements without making use of the curlometer technique. In their work they focused on the spatial distribution of energy conversion processes across the magnetopause, where the sign of $\boldsymbol{E} \cdot \boldsymbol{J}$ allows the distinction between load and dynamo processes.

The aim of our study is to add another piece to the picture of the global current distribution at Earth's magnetopause, concentrating especially on the orientation of current flows at different regions of the day-side magnetopause in order to compare it with the existing models. Because of Cluster's polar orbit we are able to intensively study the current structures at high latitudes in the vicinity of the cusp regions. 


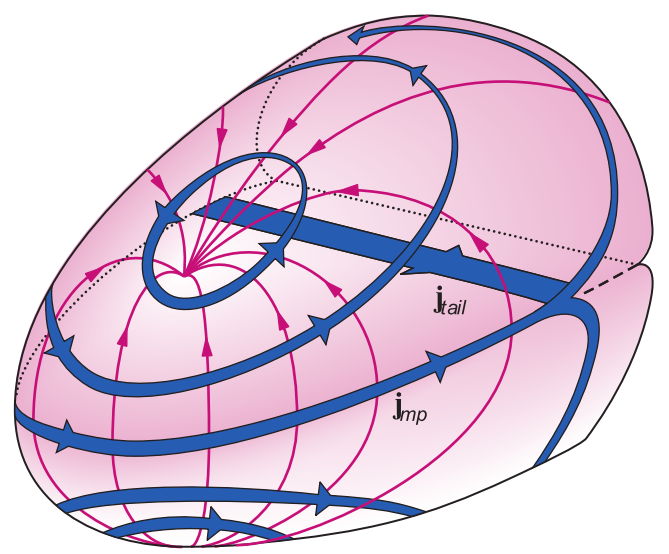

Figure 1. Three-dimensional geometry of day-side magnetopause currents. Figure courtesy of Wolfgang Baumjohann.

Using the 3-D information obtained by the four Cluster spacecraft and applying the curlometer technique allows us to directly investigate the local 3-D current densities. Hereby it is not necessary to know the actual magnetopause orientation as in cases when the current density is derived from data retrieved by fewer than four spacecraft (e.g. Dunlop et al., 2015).

To achieve an appropriate spatial resolution in terms of investigating global magnetopause current structures, we confine our event selection by Cluster configurations with interspacecraft distances no larger than about $300 \mathrm{~km}$. In addition, we perform an error analysis of the curlometer technique focussing on the special conditions in our study.

\section{The curlometer technique}

\subsection{Definition}

The curlometer analysis technique was first applied to magnetic field measurements obtained by the Cluster spacecraft by Dunlop et al. (2001). Combining 3-D magnetic field data simultaneously measured by each spacecraft, it estimates $\nabla \times \boldsymbol{B}$ and thus the local current density by calculating the difference approximation

$\mu_{0} \boldsymbol{J} \cdot\left(\Delta \boldsymbol{r}_{i j} \times \Delta \boldsymbol{r}_{i k}\right)=\Delta \boldsymbol{B}_{i j} \cdot \Delta \boldsymbol{r}_{i k}-\Delta \boldsymbol{B}_{i k} \cdot \Delta \boldsymbol{r}_{i j}$

of Ampère's law, where

$\Delta \boldsymbol{r}_{i j}=\boldsymbol{r}_{j}-\boldsymbol{r}_{i}$ and

$\Delta \boldsymbol{B}_{i j}=\boldsymbol{B}_{j}-\boldsymbol{B}_{i}, \quad i, j, k=1,2,3$

for each face $(i, j, k)$ formed by the spacecraft tetrahedron. The variable $\boldsymbol{r}_{i}$ denotes the location of spacecraft $i$ and $\boldsymbol{B}_{i}$ its magnetic field vector measurement. Calculating the current densities for three differently orientated faces allows the re-projection of the local current density into a Cartesian coordinate system.

\subsection{Error sources}

The approximation made in Eq. (1) implies the assumption of linear variations in the magnetic field across the spacecraft tetrahedron. To check to what extent this assumption is violated Dunlop et al. (1988) suggested to calculate $\nabla \cdot \boldsymbol{B}$ via

$$
\begin{aligned}
\nabla & \cdot \boldsymbol{B}\left|\Delta \boldsymbol{r}_{i j} \cdot\left(\Delta \boldsymbol{r}_{i k} \times \Delta \boldsymbol{r}_{i l}\right)\right| \\
& =\left|\sum_{j, k, l} \Delta \boldsymbol{B}_{i j} \cdot\left(\Delta \boldsymbol{r}_{i k} \times \Delta \boldsymbol{r}_{i l}\right)\right|,
\end{aligned}
$$

which becomes non-zero as a consequence of the influence of non-constant spatial gradients in the magnetic field. Nevertheless, Paschmann and Daly (1998) and Dunlop et al. (2002) used model fields to show that $\nabla \cdot \boldsymbol{B}$ does not reflect the error of the current calculation very well when the spacecraft tetrahedron or the geometry of the magnetic field is highly distorted.

Both $\nabla \times \boldsymbol{B}$ and $\nabla \cdot \boldsymbol{B}$ are sensitive to the shape of the spacecraft tetrahedron and its orientation with respect to the ambient magnetic field. As demonstrated by Paschmann and Daly (1998) the curlometer results based on tetrahedron configurations near a regular tetrahedron statistically lead to a higher accuracy of the current determination for magnetic field structures of different degrees of distortion than those based on irregular tetrahedron configurations. For this work we therefore apply the curlometer only to magnetic field data which were obtained by Cluster during configurations near a regular tetrahedron. To quantify the shape of the tetrahedron we use the one-dimensional quality factor $Q_{G}$, which is defined by

$Q_{G}=\frac{\text { True Volume }}{\text { Ideal Volume }}+\frac{\text { True Surface }}{\text { Ideal Surface }}+1$

(vom Stein et al., 1992). The ideal volume and the ideal surface denote the volume and the surface of a tetrahedron with a side length equal to the average value of the six side lengths of the true tetrahedron. $Q_{G}$ equals its maximum value of 3 when the true tetrahedron is of perfect regular shape. If all four spacecraft lie in a line, $Q_{G}$ takes the minimum value of 1. This quality factor should not be confused with the one used in Dunlop et al. (2016), which gives the value of Eq. (4) and does not contain any information about the Cluster geometry.

Of course the accuracy of the current determination by the curlometer also depends on the accuracy of the determination of $\boldsymbol{r}$ and $\boldsymbol{B}$ and on the correct timing of the measurements at all four spacecraft themselves. Laakso et al. (2009) give the measurement uncertainties of the FGM experiment as 0.1 to $0.2 \mathrm{nT}$ for low fields $(|B|<200 \mathrm{nT})$ and below $0.4 \mathrm{nT}$ for higher fields $(|B|>200 \mathrm{nT})$. The accuracy of the position determination was initially announced to meet $5 \mathrm{~km}$. During the mission and depending on the spacecraft constellation much better accuracies of some tens to hundreds of metres were 


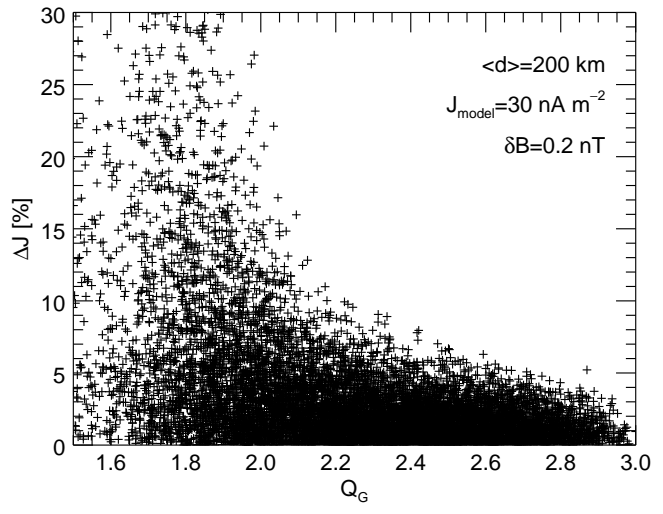

Figure 2. Relative error of the current density determination by the curlometer as a function of the tetrahedron's quality factor.

achieved. Further discussions concerning the influences of timing and measurement errors can be found, for example, in Dunlop et al. (2002).

In order to use the quality factor, $Q_{G}$, as a selection criterion for our study we conduct a brief analysis of the correlation between the error of the current density determination and the quality factor. We therefore use a model current tube which is occupied by a set of 10000 model tetrahedron configurations sensing the current's magnetic field at each tetrahedron's vertices. The current determined by the curlometer technique for every model tetrahedron is then compared to the initial current model.

We choose a cylindrical current tube with a homogeneous current density. The size of the current tube is chosen in such a way that the complete set of model tetrahedrons lies within the current tube. The tetrahedrons exhibit a random orientation with respect to the current tube and their quality factors cover values from about 1 to 3 . Measurement errors, $\delta \boldsymbol{B}$, are simulated by adding random noise to the magnetic field values.

Figures 2 and 3 display the relative error of the current density magnitude $\Delta J=\left(\left|\boldsymbol{J}_{\text {Curlometer }}\right|-\left|\boldsymbol{J}_{\text {model }}\right|\right)$. $\left|J_{\text {model }}\right|^{-1}$ and the deviation $\Delta \phi$ of the calculated current direction for a case with a model current density $\boldsymbol{J}_{\text {model }}=$ $30 \mathrm{nAm}^{-2}$, an average inter-spacecraft distance $<d>=$ $200 \mathrm{~km}$, and a noise magnitude of $\delta \boldsymbol{B}=0.2 \mathrm{nT}$. Both the error in magnitude and the error in direction show an approximately linear behaviour in the regime of $2.3<Q_{G}<2.9$, while at quality factors less then 2.2 the errors increase dramatically. For smaller sizes of the tetrahedron or the current density the errors rise as the noise value $\delta \boldsymbol{B}$ has more influence on the calculations. Our investigation showed that halving $\langle d\rangle$ or $\boldsymbol{J}_{\text {model }}$ approximately doubles $\Delta J$ and $\Delta \phi$ in the regime $Q_{G}>2.3$. Simulating the effect of an error in position determination of a worst-case value as big as $\delta \boldsymbol{r}=5 \mathrm{~km}$ has only minor effects on the results and can be neglected. Based on our results for typical conditions of the magnetopause crossing events we investigate in our study, we

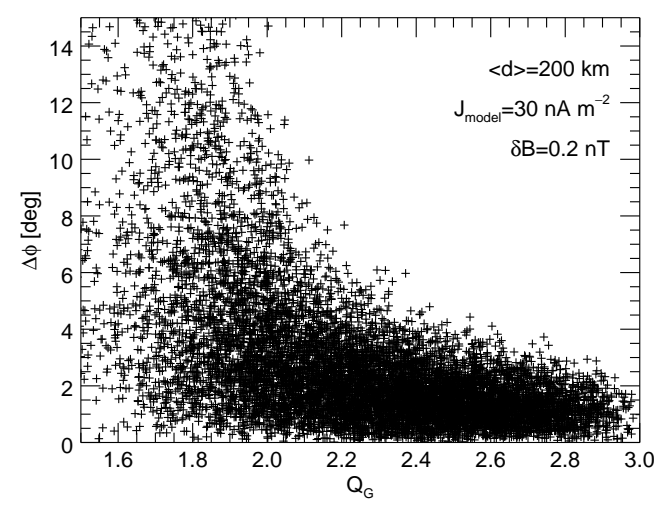

Figure 3. Deviation of the current density direction by the curlometer as a function of the tetrahedron's quality factor.

decided to choose $Q_{G} \geq 2.5$ as a data selection criterion. It allows us to expect accuracies of at least 2 to $10^{\circ}$ in direction and 3 to $15 \%$ for the relative error in magnitude.

\section{Data selection and preparation}

\subsection{Data used}

For our investigation we use Cluster magnetic field data from the fluxgate magnetometer (FGM) (Balogh et al., 2001) at spin resolution $(0.25 \mathrm{~Hz})$. Additionally, data from the Cluster Ion Spectrometry (CIS) instrument (Rème et al., 1997) are used to support the identification of magnetopause crossings. The data are retrieved from the Cluster Active Archive (Laakso et al., 2010). To sufficiently match the spatial dimensions of the magnetopause and its current flows we use data obtained while the average distance between the Cluster spacecraft was about $300 \mathrm{~km}$ or less. With this criterion we are limited to looking for day-side magnetopause transitions within the time range from February to May 2002 and from December 2003 to May 2004 where magnetic field data are available for 171 inbound and outbound orbit segments crossing the magnetopause. Because of the evolution of the Cluster tetrahedron during each orbit, several segments, mainly inbound ones, possess quality factors much lower than 2.5, where reliable curlometer results can not be expected. This leaves us 106 orbit segments suitable for our study. Figure 4 illustrates the data selection process.

\subsection{Data preparation}

Panov et al. (2008) calculated typical values of the magnetopause thickness of 500 to $3000 \mathrm{~km}$ at Cluster's highlatitude orbits used in our study. Therefore, the chosen interspacecraft distances prove to be suitable for our investigation of current features with spatial dimensions of a few hundreds of kilometres and effectively damps the influence of currents at scales up to a few tens of kilometres. 


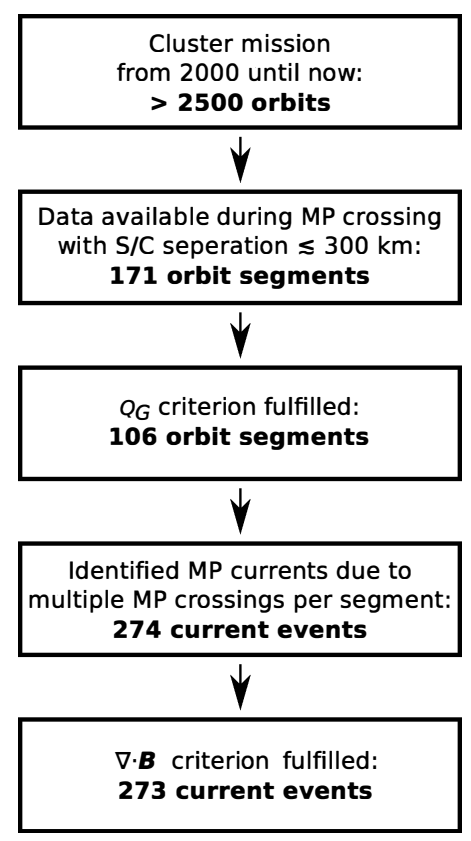

Figure 4. This illustration of the data selection process shows the shrinking number of suitable data sets. From over 2500 Cluster orbits only a few orbit segments match the spatial requirements and the quality criterion for our day-side magnetopause current investigation.

To reduce the influence of high-frequency fluctuations which are likely to lead to uncertain curlometer results (Dunlop et al., 2001) we apply a $100 \mathrm{~s}$ moving average to the $0.25 \mathrm{~Hz}$ Cluster data before calculating the current densities and identifying current events. This value is comparable to resolution limitations used in previous studies; for example, Dunlop et al. (2002) used data resolutions of 1 to $5 \mathrm{~min}$ for different events.

With a typical velocity of Cluster of about $2.5 \mathrm{~km} \mathrm{~s}^{-1}$ during magnetopause crossings investigated in this study, our chosen averaging window corresponds to a spatial averaging window of about $250 \mathrm{~km}$ along the Cluster trajectory, which is consistent with the spatial limitations due to the inter-spacecraft distances. The first two panels of Fig. 5 show the influence of the averaging window on the derived current density for an example event.

The averaging window applied to the magnetic field data smooths the four magnetic field measurement time series of each spacecraft. The curlometer tool utilizes the six differences between those four individual measurements. The averaging windows works as a low-pass filter and causes these differences to lose only high-frequency information while all other information included in the magnetic field measurements is maintained. As a result, the peak values of the calculated current density become smaller, while the average magnitude of the current components at scales of about 100 $s$ and more are not influenced significantly. The direction of

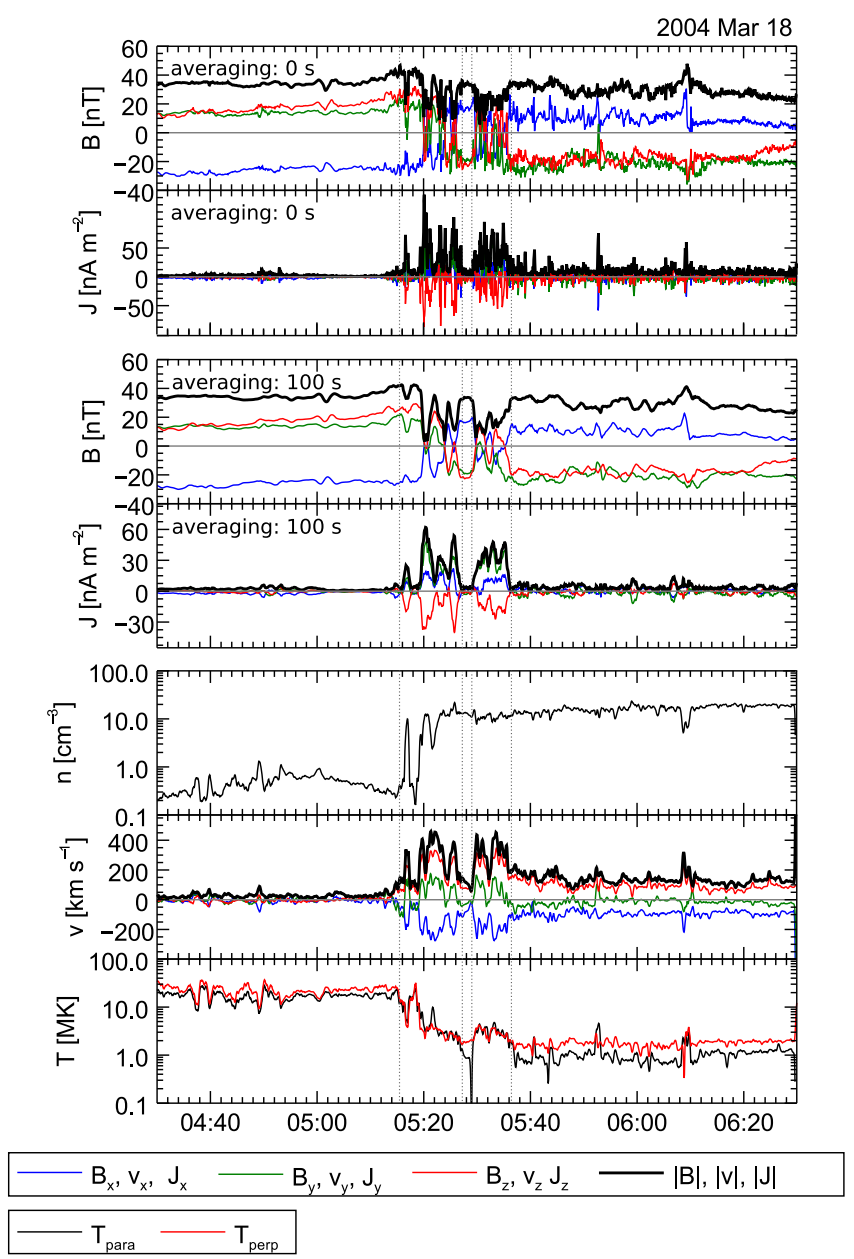

Figure 5. Outbound magnetopause crossing through the entry layer region on 18 March 2004. The upper and middle panels show the magnetic field and the curlometer result in GSE coordinates with spin resolution data and after application of a 100 s averaging window, respectively. The lower panel shows CIS hot ion particle density $n$, velocity $v$, and temperature $T$. A complete magnetopause transition between 05:16 and 05:27 UT and a second contact with the transition layer between 05:29 and 05:36 UT are visible. Both are accompanied by accelerated plasma bursts and a distortion of the magnetic field. The curlometer results shows a series of encounters with similarly orientated current layers across the transition layer.

the resulting current is less fluctuating and shows a more stable behaviour in the time series. The average direction along an identified current structure itself is influenced by the $100 \mathrm{~s}$ window only to a minor extent. Comparing the curlometer results of all current events investigated in this study with and without the application of the averaging window leads to average deviations of $6.1 \%$ in magnitude and of $2.8^{\circ}$ in angle.

For highly dynamic cases when Cluster crosses the magnetopause several times along one segment of its trajectory, multiple current signatures are merged by the $100 \mathrm{~s}$ averag- 
ing window. If the corresponding magnetic field conditions are stable for about at least $100 \mathrm{~s}$ the resulting current signature is treated as one magnetopause crossing event. Unstable conditions during highly dynamic cases are filtered out due to this preprocessing and are not included in this study.

\subsection{Magnetopause crossing identification and classification}

To identify an inner and an outer edge of the magnetopause currents for each crossing, we perform a visual inspection of every selected magnetopause transition event. First, the curlometer results are used to find the most significant current structures. In a second step we check whether those currents can be associated with the corresponding expected changes in the plasma properties monitored in the ion particle data obtained by CIS. Because of the relative movement of the magnetopause with respect to the Cluster spacecraft we can regularly identify several magnetopause crossings within one orbit segment. This enables us to enlarge our data basis for the statistical survey. A total of 274 single current events are identified, and because of a high value in $\nabla \cdot \boldsymbol{B}$ in one case 273 current events are used in our study (see Fig. 4).

Figure 5 shows an example of a magnetopause crossing during the outbound orbit branch on 18 March 2004. Cluster encounters the magnetopause twice; the first encounter between 05:16 and 05:27 UT is a complete transition through the magnetopause and the second one between 05:29 and 05:36 UT enters the transition layer partially. The relative motion of the magnetopause leads to several contacts with the current layer, as visible in the current density derived by the curlometer in the second panel of the figure.

The high-latitude Cluster orbits intersect the magnetopause roughly in the vicinity of the polar cusps. The varying formation and movement of the magnetopause, depending on the solar wind conditions, as well as the evolution of the Cluster orbits over time cause the Cluster magnetopause crossings to happen at different locations on the magnetopause. Based on the plasma properties along the Cluster trajectory it is possible to classify the crossing events with respect to different regions at the magnetopause.

In our study we divide all crossing events into the following classes: low-latitude-like boundary (LL), entry layer (EL), cusp (C), and plasma mantle (PM) transition. Figure 6 shows the location of these regions at the magnetopause. Transitions showing signatures that made it difficult to distinguish between an entry layer and a cusp transition are ascribed to a fifth group: $\mathrm{EL}+\mathrm{C}$.

The low-latitude-like boundary transitions are located on the magnetopause sunward of the cusp region (Haerendel et al., 1978). Characteristic for the low-latitude-like boundary transition is a significant drop in the ion density and the ion velocity accompanied by an increase in temperature (e.g. Haerendel et al., 1978; Eastman and Hones Jr., 1979). It separates the cold magnetosheath plasma from the hot and thin

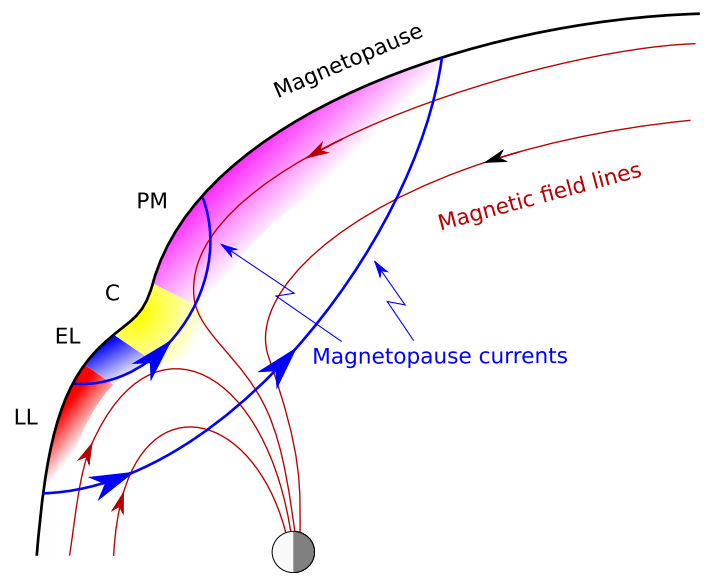

Figure 6. Illustration of the location of the different regions used to classify the magnetopause crossing events. Red denotes the lowlatitude-like boundary (LL), blue the entry layer (EL), yellow the cusp (C), and magenta the plasma mantle (PM) transitions. The current flow along nested loops around the cusp is pictured with blue lines.

magnetospheric plasma. In most cases the low-latitude-like boundary also shows a clear change in the orientation of the magnetic field. Adjacent to the low-latitude-like boundary layer and the cusp the entry layer represents a region where magnetosheath plasma is injected into the magnetosphere (e.g. Frank, 1971; Haerendel and Paschmann, 1975; Haerendel et al., 1978). The changes in plasma density and temperature are similar to those of low-latitude-like transitions. In addition, the entry layer can be characterized by bursts of accelerated sheath plasma that exceeds the flow velocity of the magnetosheath (Panov et al., 2008). During these plasma bursts, the magnetic field often undergoes rapid fluctuations as well as drops in magnitude. Figure 5 displays a typical signature of a transition through the entry layer.

At the cusp region, the plasma flow is intermittent, sometimes stagnant, and the temperature is raised compared to the sheath and lobe plasma (e.g. Panov et al., 2008). Particle energy spectrograms show isotropic and broadened energy distributions during cusp transitions (e.g. Smith and Lockwood, 1996; Rème et al., 2001). The magnetic field is fluctuating and often drops to very low magnitudes during a cusp transition (e.g. Cargill et al., 2001). Plasma mantle transitions are located at the high-latitude magnetopause tail-ward of the cusp region. They can be identified by magnetosheathlike plasma within the transition region and in the adjacent plasma of the magnetospheric lobes. The plasma shows a relatively steady tail-ward plasma flow and a significant temperature anisotropy (e.g. Rosenbauer et al., 1975; Haerendel et al., 1978). The density lies in between those found in the magnetospheric lobe plasma and sheath plasma. 


\section{Reference magnetopause}

Because magnetosphere's scaling depends on the varying solar wind conditions the intersection of the Cluster orbits with the magnetopause scatter significantly when displayed in a geocentric solar ecliptic (GSE) coordinate system. To use a common frame of reference for all events investigated in our study we introduce a reference magnetopause. For simplification, we use a second-order approximation, i.e. a paraboloidal magnetopause shape, which is valid as we are not considering the far tail regions in our study. Following Nabert et al. (2013) we use the parametrization

$x=\Delta_{\mathrm{MP}}-\sum_{t=y, z} c_{\mathrm{MP}, t} t^{2}$.

$\Delta_{\text {MP }}$ depicts the sub-solar magnetopause stand-off distance with respect to the centre of the Earth (see Fig. 7). The geometric parameters $c_{\mathrm{MP}, t}$ represent the magnetopause curvature in $t=y$ and $t=z$ direction. Nabert et al. (2013) deduce values of

$c_{\mathrm{MP}, y}=0.4 \frac{1}{\Delta_{\mathrm{MP}}}, \quad c_{\mathrm{MP}, z}=0.5 \frac{1}{\Delta_{\mathrm{MP}}}$

from an analytical zeroth-order approach solving the MHD equations in the magnetosheath.

For each identified current the mean value of the tetrahedron barycentre's position vector is calculated. By radial projection along the Earth-spacecraft line the intersection of this vector with the reference magnetopause is calculated (compare Fig. 7).

\section{Results}

\subsection{Directions of magnetopause currents}

The 273 magnetopause currents investigated in our study allocate into our five classes as follows: 75 low-latitudelike transitions, 66 entry layer transitions, 48 events showing characteristics of both entry layer and cusp transitions, 53 cusp transitions and 31 plasma mantle transitions.

Figures 8 to 10 show the normalized $x-y$ component and $y-z$ component of all currents calculated by the curlometer for each class. The circles depict the position of each magnetopause crossing on the reference magnetopause projected into the $x-y$ plane and the $y-z$ plane of a GSE coordinate system. The arrows of normalized length represent the direction of the current flow within these planes. The colour gives information about the $x$ component of each current: green and red indicate that the currents point towards the Sun and towards the Earth, respectively. The angle between the current and the $x$ axis is depicted in the legend. Currents that are shown in black are flowing nearly parallel to the $y-z$ plane. The lighter the colours the more perpendicular the current flows with respect to the $y-z$ plane. The bottom panel of the

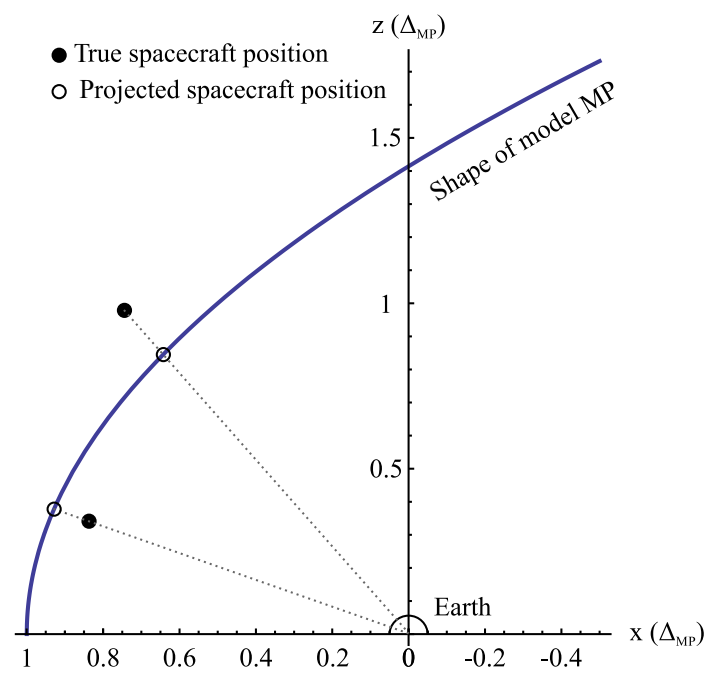

Figure 7. Illustration of the model magnetopause used as a reference magnetopause for data presentation. The dotted lines show example projections of two different currents from their true location to the location at the reference magnetopause.

figures additionally show the occurrence rate of the current angle within the $y-z$ plane with respect to the positive $y$ axis.

As visible in the figures a distinction of low-latitude-like, entry layer, cusp and plasma mantle transitions based purely on the crossing's location on the chosen reference magnetopause would not be possible as the circles cover roughly the same areas for all pictured classes.

Of the 75 currents in the LL case (Fig. 8), 63 exhibit a positive $y$ component. This corresponds well to the classical model of the day-side Chapman-Ferraro current flowing from dawn to dusk at low latitudes as illustrated in Fig. 1. The colour code shows that on the dawn side $(y<0)$ of the magnetopause the currents dominantly point towards the Sun, whereas the dusk side $(y>0)$ is dominated by currents pointing earthward. This also conforms to the magnetopause's elliptical shape, leading to sunward current flows at dawn and earthward current flows at dusk along the magnetopause.

In the EL case, 13 of the investigated currents are pointing dawn-ward and 53 are pointed dusk-ward. All the currents on the dawn side point towards the Sun or flow nearly parallel to the $y-z$ plane. On the dusk side again the opposite is the case. The curlometer results of LL and EL cases show qualitatively the same behaviour regarding the $J_{y}$ and $J_{z}$ components. In contrast to the LL case the $J_{z}$ component is more significant for EL transitions. Interestingly, only one magnetopause crossings on the southern hemisphere has been assigned to the EL class due to the plasma properties. Twelve currents associated with the entry layer transitions exhibit negative $z$ components. In the picture of nested current rings around the cusp region as illustrated in Figs. 1 and 6 for currents just below the cusp on the day-side magnetopause one would expect negative $z$ - and positive $x$ components at the dawn side 

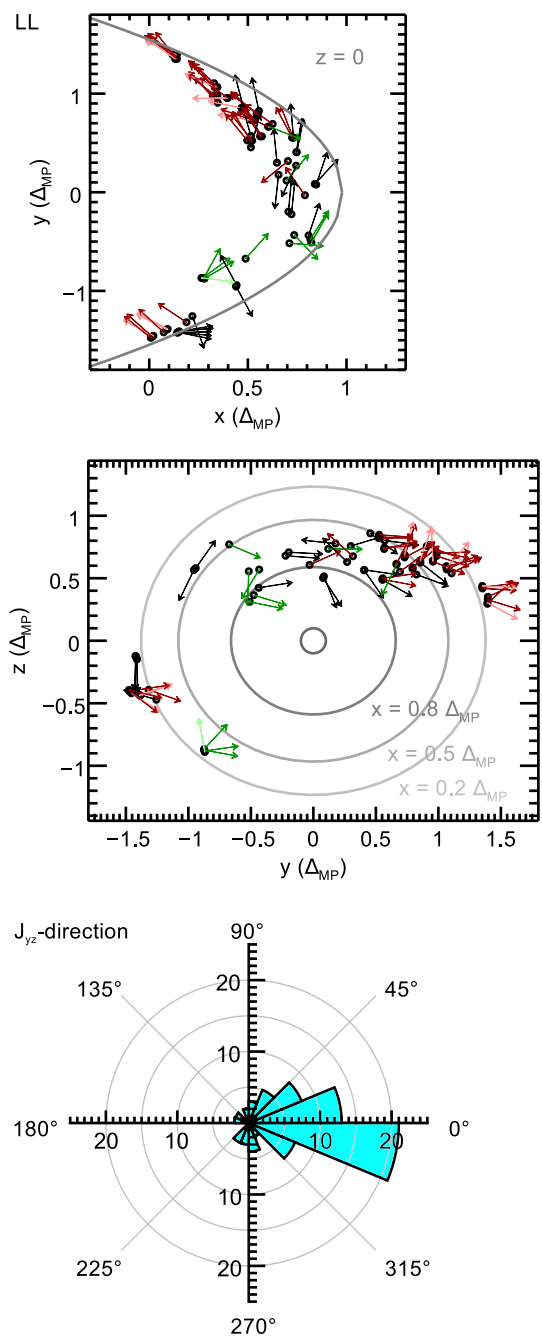
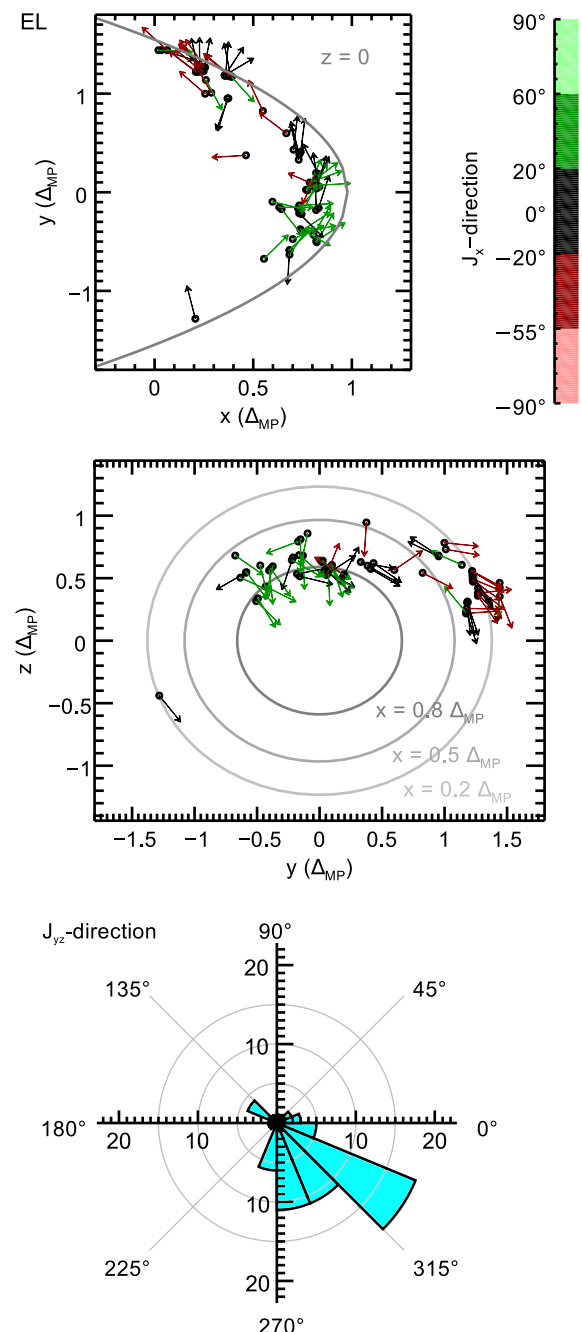

Figure 8. Current directions for LL (left) and EL (right) transitions in GSE coordinates at the reference magnetopause projected into the $x-y$ plane (top panel) and $y-z$ plane (middle panel). The grey paraboloid and ellipsoids represent the magnetopause position at $z=0$ and $x=0.2,0.5,0.8 \Delta_{\mathrm{MP}}$, respectively. The colour code represents the $J_{x}$ direction. The polar histograms show the occurrence rate of the current angle within the $y-z$ plane with respect to the positive $y$ axis.

and just the opposite, positive $z$ and negative $x$ component, at the dusk side. The current directions from the curlometer determined for the EL class are not in agreement with this model. However, as the origin of the day-side entry layer is thought to be the process of day-side reconnection (Haerendel et al., 1978) it is quite probable that the magnetopause's shape does not equal the smooth geometry of a quiet and simplified magnetopause at this region.

We assigned 48 currents to the EL $+\mathrm{C}$ class (Fig. 9) for cases showing entry layer as well as cusp transition characteristics. The events are concentrated within a range of $\pm 1 \Delta_{\mathrm{MP}}$ from the $x-y$ plane. Compared to the LL and EL case we find a turn of the current flow with respect to the $J_{y}$ and the $J_{z}$ component, clearly visible in the histogram of Fig. 9. Twenty-six of the EL $+\mathrm{C}$ currents possess a duskdawn orientation and 22 a dawn-dusk orientation. The num- bers are the same for northward and southward directions. The distribution of the $J_{x}$ component is also almost even, with 25 currents pointing towards the Sun and 23 towards the Earth. As visible by the number of black arrows in Fig. 9 the $J_{x}$ component exhibits a decreased magnitude compared to LL and EL transitions.

The $\mathrm{C}$ case (Fig. 10, left) with 53 currents used in this study shows a growing ratio of $36 / 17$ of dusk-dawn to dawn-dusk orientations clearly marking the change in the current direction between low-latitude and high-latitude regions on the day-side magnetopause. Like in the $\mathrm{EL}+\mathrm{C}$ case several currents are more parallel to the $y-z$ plane compared to the LL and EL currents.

With 31 currents in the PM class (Fig. 10, right) the minority of the investigated current structures are located at the high-latitude magnetopause on the night side of the cusp. 

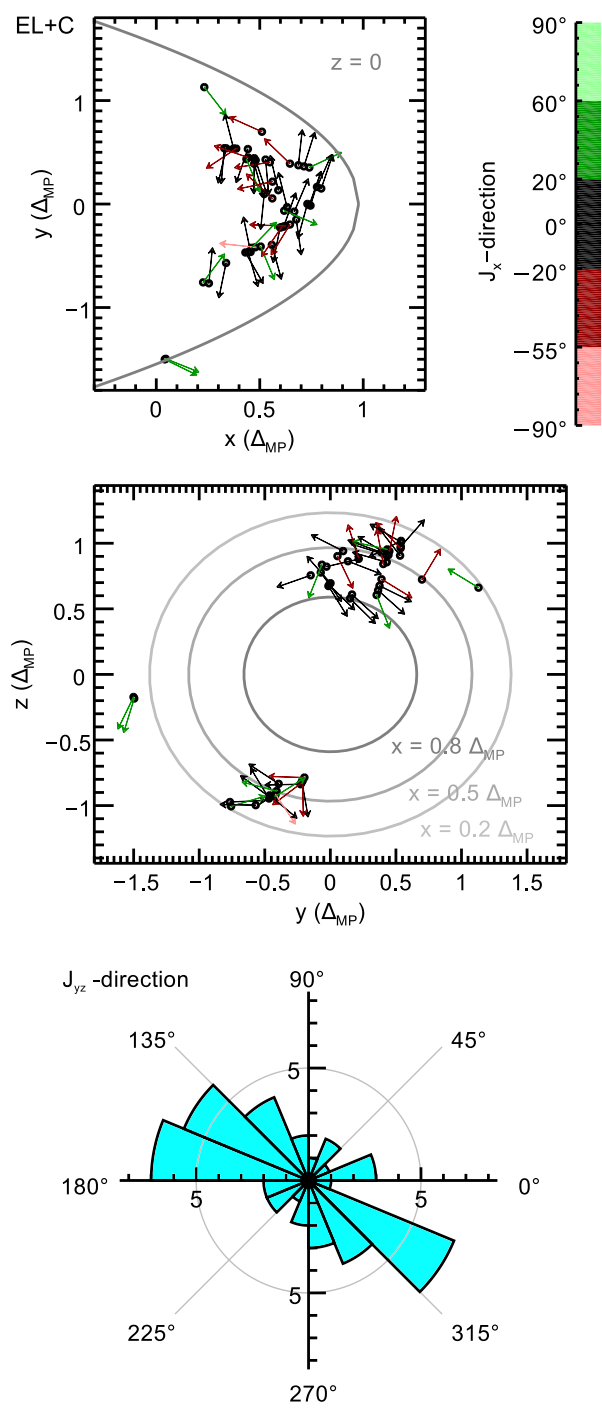

Figure 9. Same as Fig. 8 but for EL $+\mathrm{C}$ transitions.

From models a current flow from dusk to dawn is expected in this region. The results of our study agree with this quite well, as visible in the histogram of Fig. 10. In addition the current's $J_{z}$ components fit well to the magnetopause's draped shape around the Earth. On the dawn side most of the currents of the southern hemisphere are pointing towards north and those at the northern hemisphere are pointing towards south. The PM transitions also shows the narrowest $J_{x}$-distribution of all five classes. In total, 22 of 31 currents exhibit angles with respect to the $x$ axis of less than $20^{\circ}$.

\subsection{Dependence on solar wind conditions}

We use 5 min averaged OMNI data which are already timeshifted to the bow shock nose prior to each current event in order to investigate dependencies on the solar wind conditions. OMNI data are available for 270 of the current events. The average magnitude of the interplanetary magnetic field
(IMF) is $5.98 \mathrm{nT}$. The IMF is directed southward in 160 cases and northward in 110 cases. Figure 11 shows the polar histograms of the $J_{y z}$ directions for IMF $B_{z}<0$ and $B_{z}>0$ separately. Independently from the magnetopause region the currents observed during northward IMF possess a wider distribution in direction than during southward IMF. In both cases LL and EL currents are dominantly orientated in the dawn-dusk direction and C and PM currents in the duskdawn direction. Only the currents ascribed to the $\mathrm{EL}+\mathrm{C}$ case show a clearly different orientation depending on the solar wind $B_{z}$ component. For southward IMF the dawndusk orientation is maintained by 13 out of 22 currents. For northward IMF 15 out of 26 currents possess a dusk-dawn orientation. This indicates that the overall observed change in direction of the magnetopause currents at the vicinity of entry layer and cusp contains a dependency from the IMF $B_{z}$ component.

In MHD simulations Lopez et al. (2011) showed that a dawn-dusk orientated current tail-ward of the cusp arises in the case of a strong southward IMF with $B_{z}=-20 \mathrm{nT}$. In our study we only find four events at the PM region with $B_{z}<$ $-6 \mathrm{nT}$ and the smallest value of $B_{z}$ is $-8.8 \mathrm{nT}$. Those four events correspond to nominal solar wind conditions and they possess currents with negative $J_{y}$ components. Hence, we are not able to observe any high-latitude magnetopause currents during IMF conditions that would allow us a comparison of the curlometer results with the simulation results by Lopez et al. (2011).

\subsection{Location of currents with respect to the plasma regimes}

Regarding the particle density, velocity, and temperature data in the example presented in Fig. 5 one can identify three different plasma regimes: the day-side magnetosphere plasma on the left-hand side until 05:16 UT and between 05:27 and 05:29 UT, the magnetosheath plasma on the righthand side from 05:36 UT, and the transition region during 05:16 to 05:27 UT, when Cluster traverses the magnetopause, and 05:29 to 05:36 UT, when the magnetopause movement causes a contact with the magnetopause. The curlometer results in the second panel of Fig. 5 show that the current structures are observed while Cluster is situated within the transition regime in this example.

In our survey we find that the location of magnetopause current structures varies with respect to the location of the outer edge of changing plasma properties for different magnetopause encounters. We distinguish the following four categories as shown in Fig. 12. (A) The current onset lies within the magnetosheath plasma and ends within $100 \mathrm{~km}$ radial distance from the outer edge of plasma changes. (B) The current structure spans across the location of the outermost plasma property changes, beginning in the magnetosheath end ending within the transition regime. (C) The current onset lies entirely within the transition regime and within $100 \mathrm{~km}$ from 

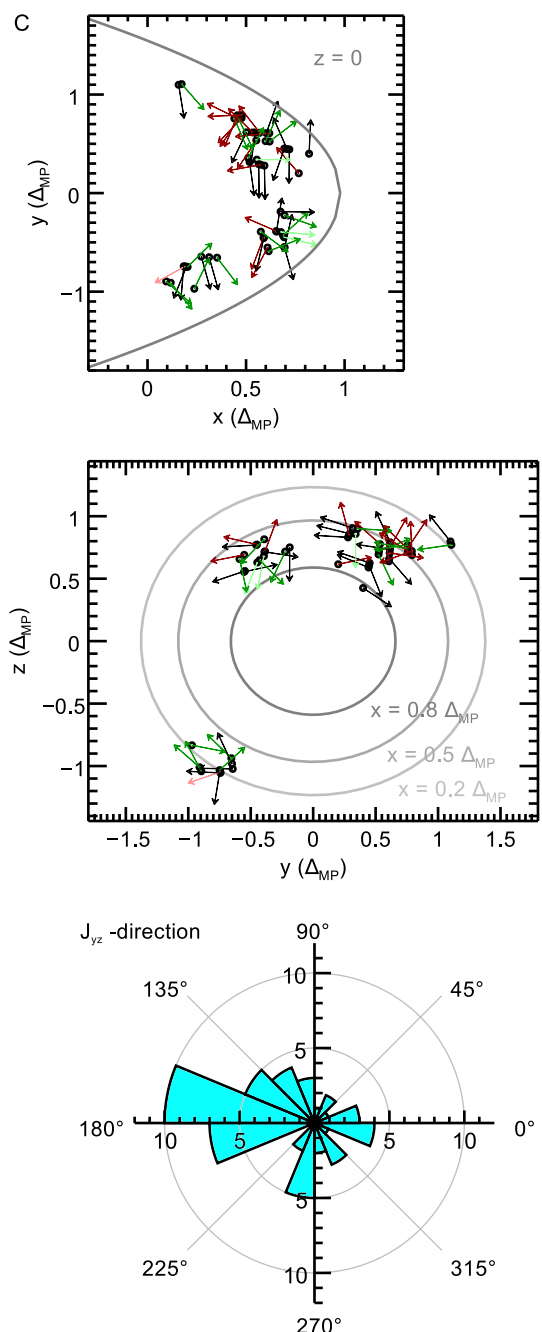

Figure 10. Same as Fig. 8 but for C (left) and PM (right) transitions.

the location of the outermost plasma changes. (D) The current structure lies within the transition regime but is detached from the outer edge of the plasma changes. A case where the current lies beyond the transition regime within the magnetosphere plasma does not appear in our study.

Counting the occurrence rates of the location categories shows that the distributions are similar for the LL and the EL case as well as for the EL $+\mathrm{C}$ and the $\mathrm{C}$ case. Therefore those cases are summarized in Fig. 13, depicting the rates for all investigated magnetopause crossings.

For magnetopause currents observed at LL and EL most of the currents, 111 out of 141 are located across or adjacent to the outermost edge of plasma changes. This result also holds for the PM case, where 23 of 31 currents are found in direct vicinity of the location where plasma changes are first observed. In contrast to this the distribution for $\mathrm{EL}+\mathrm{C}$ and $\mathrm{C}$ cases shows a significant number, 79 of 101, of completely detached current structures. Once again this fits with the idea
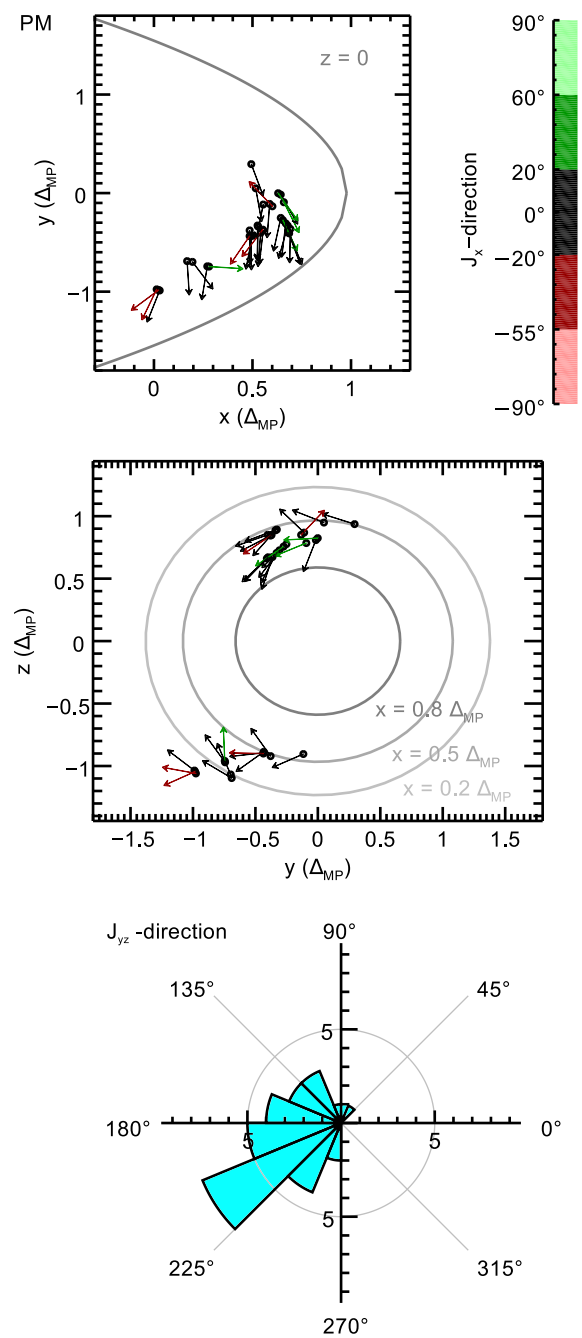

of a turbulent cusp region, where the formation of a clear and steady magnetopause current is unlikely.

Overall we count 48 current structures that are located in front of the transition regime identified by the changes in plasma properties (category A) and 127 currents clearly detached (category D). As indicated by the colours in Fig. 13 our survey shows no clear evidence for an influence of the IMF orientation on the location of the currents. Checking for a dependence on the IMF magnitude leads to the same result.

\subsection{Current magnitudes}

For better visibility the current directions in Figs. 8, 9 and 10 are displayed with normalized magnitudes. A statistic of the true averaged magnitudes for all observed current events is presented in Fig. 14. As northward and southward IMF does not influence the distribution in a significant way we omit the indication of the IMF $B_{z}$ component in this depiction. With 182 out of 273 , the majority of the evaluated cur- 


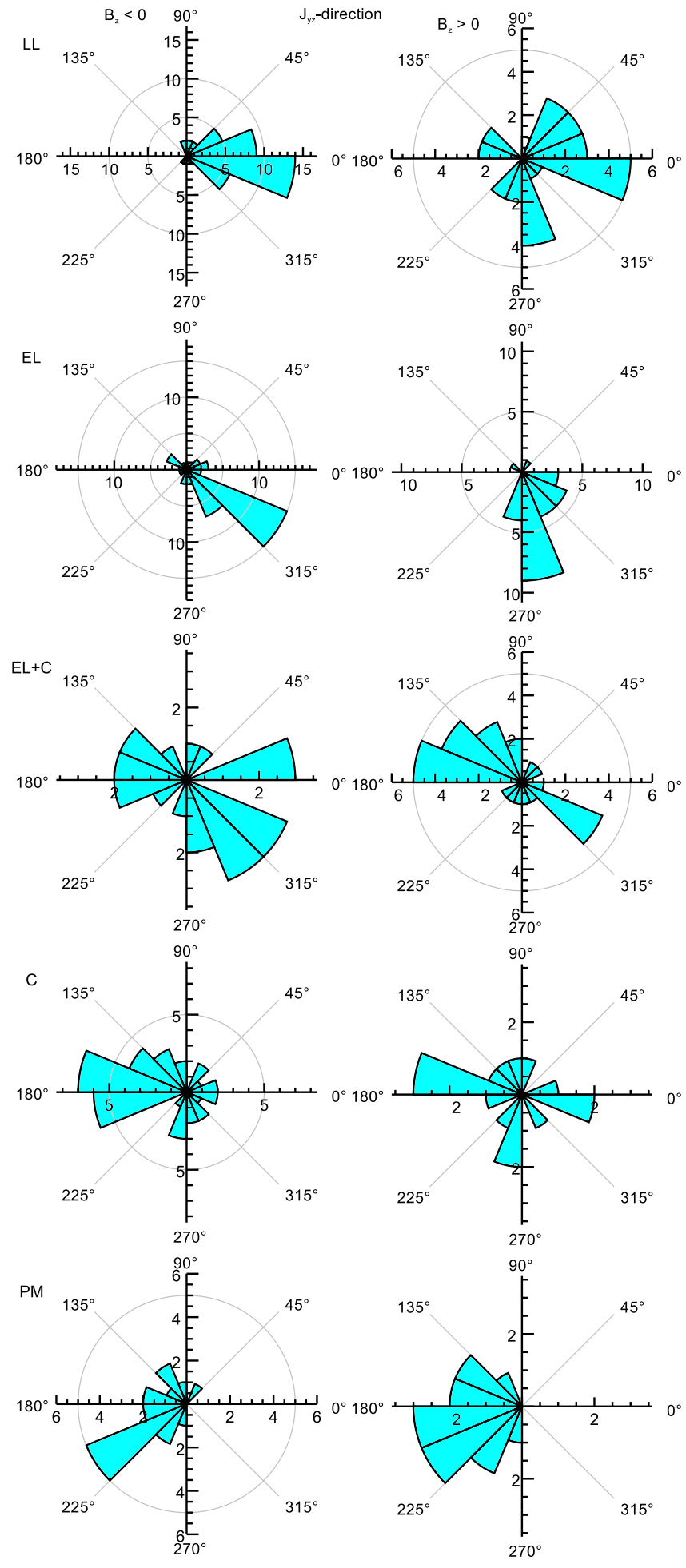

Figure 11. Occurrence rate of the current angle within the $y-z$ plane with respect to the positive $y$ axis during southward (left) and northward (right) IMF for LL, EL, EL + C, C, and PM transitions (from top to bottom).

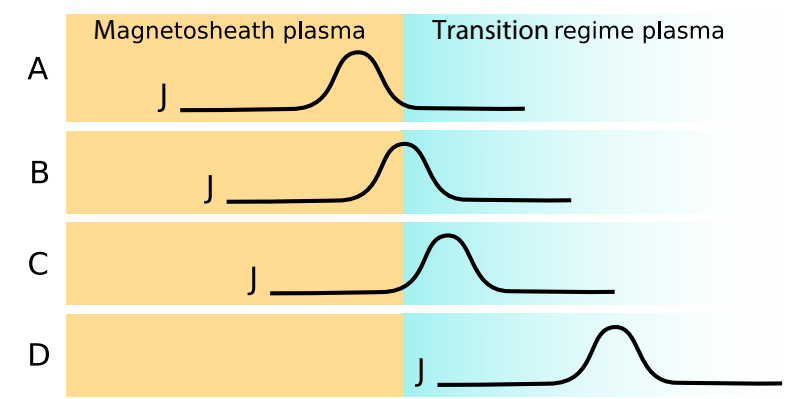

Figure 12. Schematic representation of the categorization of the current locations with respect to the ambient plasma regimes. The outermost edge, where changes in the plasma can be observed, is represented by the changing background colour. The four cases show currents that lie in front of (A), across (B), shortly behind (C) and clearly detached from (D) this edge.

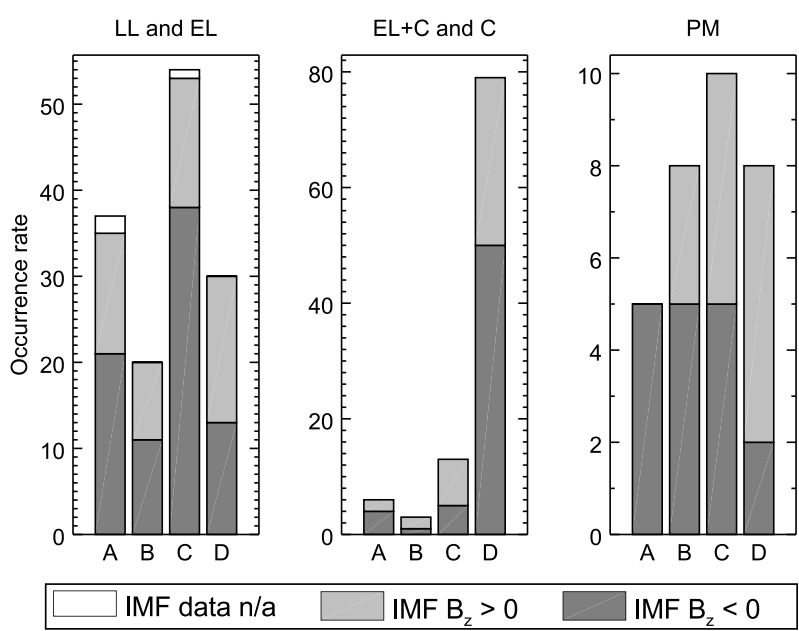

Figure 13. Occurrence rate of the current locations for the different transition regions as described in the text and by Fig. 12 .

rents possess averaged magnitudes of 5 to $20 \mathrm{nA} \mathrm{m}^{-2}$ independently of the magnetopause region they are observed at. For larger magnitudes of the IMF (lower panel) the current magnitudes are increased. This can be explained by the enhanced magnetic pressure of magnetosheath plasma, which scales with $p_{\text {mag }} \sim|B|^{2}$ and the increased compression of the magnetosphere. Average current densities between 30 and $85 \mathrm{nA} \mathrm{m}^{-2}$ regularly appear especially for $\mathrm{C}$ and $\mathrm{PM}$ cases during stronger IMF. A similar result was reported by Panov et al. (2008), who evaluated the peak current density values for 52 magnetopause crossings, showing that PM current peak magnitudes were about 2 times larger than those at the LL region.

\section{Conclusions}

Applying the presented selection criteria to the available Cluster data allows us to investigate 273 current events with 


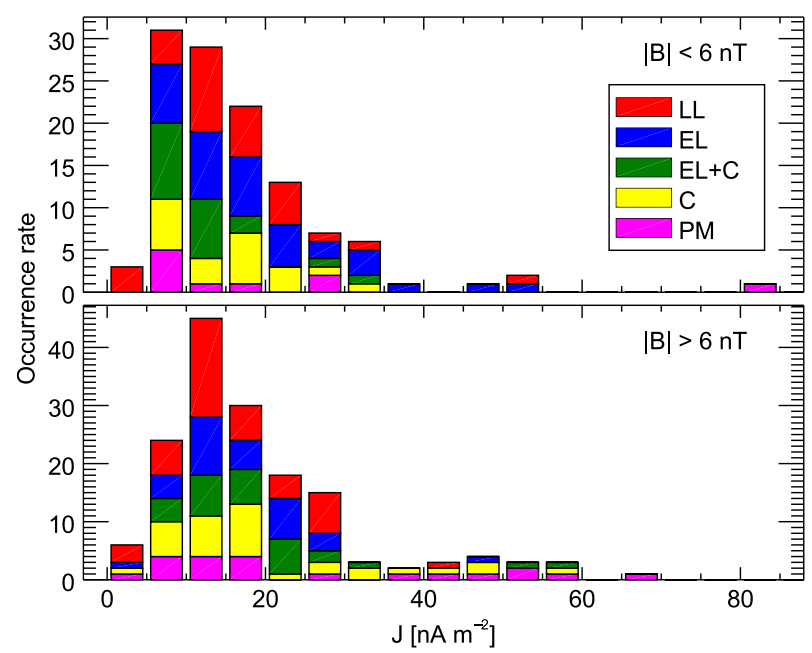

Figure 14. Occurrence rate of current density magnitudes averaged across each current event for solar wind magnetic field magnitudes smaller (upper panel) and greater (lower panel) than $6 \mathrm{nT}$.

average inter-spacecraft distances of about $300 \mathrm{~km}$ and less. The results of our survey, based on the usage of the curlometer technique, show that the magnetopause current flow directions of these current events match expectations based on existing magnetopause current models and MHD simulations. We find dawn-dusk-orientated currents on the day side of the cusp and dusk-dawn-orientated currents at high latitudes on the night side of the cusp. Following the ellipsoid-like shape of the magnetopause, the low-latitude currents point towards the Sun at dawn and towards Earth at dusk, whereas the highlatitude currents flow towards lower latitudes at the side of the magnetopause. The majority of the investigated currents around the cusp region confirm the picture of closed current loops.

In cases of entry layer, entry layer plus cusp, and cusp transitions that lie in between the low-latitude-like boundary and the plasma mantle transition region, we find gradually changing current direction and a wider distribution in direction that can be explained by the turbulent local boundary leading to an unstable formation of the magnetopause with variable topology (e.g. Haerendel et al., 1978; Savin et al., 2001).

The current directions observed in the entry layer plus cusp case show a dependence on the IMF $B_{z}$ component. In the other four cases, the current direction of the majority of the currents is not influence by the IMF orientation but the current directions are distributed significantly wider during northward than during southward IMF.

The comparison of the location of the currents with the outermost edge of changes in the plasma environment shows that the current structures lie in front of, across, or behind the edge of the transition regime. For the cusp and the entry layer plus cusp cases most of the currents are completely detached from the outer edge of this regime. At the other re- gions of the magnetopause the majority of the currents lie in the close vicinity of this edge as one would expect for cases of a clearly formed boundary. In several of theses cases the current features are observed slightly but clearly in front of the change in the plasma properties. The location of the current with respect to the plasma environment is shown to be independent of IMF orientation and magnitude.

Calculating the averaged current density magnitudes for all current events shows typical values of 5 to $20 \mathrm{nA} \mathrm{m}^{-2}$. Average current magnitudes are slightly higher during higher IMF magnitudes. Some currents at the entry layer plus cusp and the plasma mantle region possess larger current densities up to $85 \mathrm{nA} \mathrm{m}^{-2}$, especially for IMF magnitudes $|B|>6 \mathrm{nT}$. This result is in accordance with earlier similar investigations of the peak current magnitudes at magnetopause crossings on the day and night side of the cusp by Panov et al. (2008).

Data availability. All Cluster data used in this study can be retrieved from the Cluster Active Archive (http://caa.estec.esa.int/ caa/). OMNI IMF data are retrieved from the Coordinated Data Analysis Web (CDAWeb) (https://cdaweb.sci.gsfc.nasa.gov/index. $\mathrm{html} /$ ).

Competing interests. The authors declare that they have no conflict of interest.

Acknowledgements. This work was financially supported by the Deutsches Zentrum für Luft- und Raumfahrt under contract 20 50OC1402. The authors thank the Cluster FGM and CIS teams and the Cluster Active Archive for processing and providing the Cluster data and J. H. King and N. Papatashvilli at AdnetSystems, NASA GSFC, and CDAWeb for providing the OMNI data. Thanks to Wolfgang Baumjohann for providing Fig. 1.

The topical editor, M. Palmroth, thanks R. E. Lopez and two anonymous referees for help in evaluating this paper.

\section{References}

Anekallu, C. R., Palmroth, M., Koskinen, H. E. J., Lucek, E., and Dandouras, I.: Spatial variation of energy conversion at the Earth's magnetopause: Statistics from Cluster observations, J. Geophys. Res.-Space, 118, 1948-1959, doi:10.1002/jgra.50233, 2013.

Balogh, A., Carr, C. M., Acuña, M. H., Dunlop, M. W., Beek, T. J., Brown, P., Fornacon, K.-H., Georgescu, E., Glassmeier, K.H., Harris, J., Musmann, G., Oddy, T., and Schwingenschuh, K.: The Cluster Magnetic Field Investigation: overview of in-flight performance and initial results, Ann. Geophys., 19, 1207-1217, doi:10.5194/angeo-19-1207-2001, 2001.

Cargill, P. J., Dunlop, M. W., Balogh, A., and the FGM team: First Cluster results of the magnetic field structure of the mid- and high-altitude cusps, Ann. Geophys., 19, 1533-1543, doi:10.5194/angeo-19-1533-2001, 2001. 
Cluster Active Archive: FGM fluxgate magnetometer - Magnetic field, spin resultion; CIS ion spectronometer - Ion Moments (High and Low Sensitivity), http://caa.estec.esa.int/caa/, last access: 14 April 2017.

Coordinated Data Analysis Web: OMNI HRO 5MIN - Alfvenic mach number, https://cdaweb.sci.gsfc.nasa.gov/index.html/, last access: 14 April 2017.

Dunlop, M. W. and Balogh, A.: Magnetopause current as seen by Cluster, Ann. Geophys., 23, 901-907, doi:10.5194/angeo-23901-2005, 2005.

Dunlop, M. W., Southwood, D. J., Glassmeier, K.-H., and Neubauer, F. M.: Analysis of multipoint magnetometer data, Adv. Space Res., 8, 273-277, doi:10.1016/0273-1177(88)90141X, 1988 .

Dunlop, M. W., Balogh, A., and Glassmeier, K.-H.: First Application of Multi-Point Magnetic Field Analysis Techniques: the Curlometer and the Discontinuity Analyser, in: Sheffield Space Plasma Meeting: Multipoint Measurements versus Theory, edited by: Warmbein, B., Vol. 492 of ESA Special Publication, p. 3, 2001.

Dunlop, M. W., Balogh, A., Glassmeier, K.-H., and Robert, P.: Four-point Cluster application of magnetic field analysis tools: The Curlometer, J. Geophys. Res.-Space, 107, 1384, doi:10.1029/2001JA005088, 2002.

Dunlop, M. W., Yang, Y.-Y., Yang, J.-Y., Lühr, H., Shen, C., Olsen, N., Ritter, P., Zhang, Q.-H., Cao, J.-B., Fu, H.-S., and Haagmans, R.: Multispacecraft current estimates at swarm, J. Geophys. Res.Space, 120, 8307-8316, doi:10.1002/2015JA021707, 2015.

Dunlop, M. W., Haaland, S., Escoubet, P. C., and Dong, X.C.: Commentary on accessing 3-D currents in space: Experiences from Cluster, J. Geophys. Res.-Space, 121, 7881-7886, doi:10.1002/2016JA022668, 2016.

Eastman, T. E. and Hones Jr., E. W.: Characteristics of the magnetospheric boundary layer and magnetopause layer as observed by Imp 6, J. Geophys. Res.-Space, 84, 2019-2028, doi:10.1029/JA084iA05p02019, 1979.

Escoubet, C. P., Fehringer, M., and Goldstein, M.: Introduction The Cluster mission, Ann. Geophys., 19, 1197-1200, doi:10.5194/angeo-19-1197-2001, 2001.

Frank, L. A.: Plasma in the Earth's polar magnetosphere, J. Geophys. Res.-Space, 76, 5202, doi:10.1029/JA076i022p05202, 1971.

Haerendel, G. and Paschmann, G.: Entry of solar wind plasma into the magnetosphere, in: Physics of the Hot Plasma in the Magnetosphere, edited by: Hultqvist, B. and Stenflo, L., 23-43, 1975.

Haerendel, G., Paschmann, G., Sckopke, N., and Rosenbauer, H.: The frontside boundary layer of the magnetosphere and the problem of reconnection, J. Geophys. Res., 83, 3195-3216, doi:10.1029/JA083iA07p03195, 1978.

Laakso, H., Taylor, M., and Escoubet, C. P.: The Cluster Active Archive - Studying the Earth's Space Plasma Environment, Edn. 2010, Springer Science \& Business Media, Berlin Heidelberg, 2009.

Laakso, H., Perry, C., McCaffrey, S., Herment, D., Allen, A. J., Harvey, C. C., Escoubet, C. P., Gruenberger, C., Taylor, M. G. G. T., and Turner, R.: Cluster Active Archive: Overview, Astrophysics Space, 11, 3-37, doi:10.1007/978-90-481-3499-1_1, 2010.
Lopez, R. E., Merkin, V. G., and Lyon, J. G.: The role of the bow shock in solar wind-magnetosphere coupling, Ann. Geophys., 29, 1129-1135, doi:10.5194/angeo-29-1129-2011, 2011.

Nabert, C., Glassmeier, K.-H., and Plaschke, F.: A new method for solving the MHD equations in the magnetosheath, Ann. Geophys., 31, 419-437, doi:10.5194/angeo-31-419-2013, 2013.

Panov, E. V., Büchner, J., Fränz, M., Korth, A., Khotyaintsev, Y., Nikutowski, B., Savin, S., Fornaçon, K.-H., Dandouras, I., and Rème, H.: CLUSTER spacecraft observation of a thin current sheet at the Earth's magnetopause, Adv. Space Res., 37, 13631372, doi:10.1016/j.asr.2005.08.024, 2006.

Panov, E. V., Büchner, J., Fränz, M., Korth, A., Savin, S. P., Rème, H., and Fornaçon, K.-H.: High-latitude Earth's magnetopause outside the cusp: Cluster observations, J. Geophys. Res.-Space, 113, A01220, doi:10.1029/2006JA012123, 2008.

Paschmann, G. and Daly, P. W.: Analysis Methods for MultiSpacecraft Data. ISSI Scientific Reports Series SR-001, ESA/ISSI, Vol. 1. ISBN 1608-280X, 1998, ISSI Scientific Reports Series, 1, 1998.

Rème, H., Bosqued, J. M., Sauvaud, J. A., Cros, A., Dandouras, J., Aoustin, C., Bouyssou, J., Camus, T., Cuvilo, J., Martz, C., Medale, J. L., Perrier, H., Romefort, D., Rouzaud, J., D’Uston, C., Mobius, E., Crocker, K., Granoff, M., Kistler, L. M., Popecki, M., Hovestadt, D., Klecker, B., Paschmann, G., Scholer, M., Carlson, C. W., Curtis, D. W., Lin, R. P., McFadden, J. P., Formisano, V., Amata, E., Bavassano-Cattaneo, M. B., Baldetti, P., Belluci, G., Bruno, R., Chionchio, G., di Lellis, A., Shelley, E. G., Ghielmetti, A. G., Lennartsson, W., Korth, A., Rosenbauer, H., Lundin, R., Olsen, S., Parks, G. K., McCarthy, M., and Balsiger, H.: The Cluster Ion Spectrometry (cis) Experiment, Space Sci. Rev., 79, 303-350, doi:10.1023/A:1004929816409, 1997.

Rème, H., Aoustin, C., Bosqued, J. M., Dandouras, I., Lavraud, B., Sauvaud, J. A., Barthe, A., Bouyssou, J., Camus, Th., Coeur-Joly, O., Cros, A., Cuvilo, J., Ducay, F., Garbarowitz, Y., Medale, J. L., Penou, E., Perrier, H., Romefort, D., Rouzaud, J., Vallat, C., Alcaydé, D., Jacquey, C., Mazelle, C., d'Uston, C., Möbius, E., Kistler, L. M., Crocker, K., Granoff, M., Mouikis, C., Popecki, M., Vosbury, M., Klecker, B., Hovestadt, D., Kucharek, H., Kuenneth, E., Paschmann, G., Scholer, M., Sckopke, N., Seidenschwang, E., Carlson, C. W., Curtis, D. W., Ingraham, C., Lin, R. P., McFadden, J. P., Parks, G. K., Phan, T., Formisano, V., Amata, E., Bavassano-Cattaneo, M. B., Baldetti, P., Bruno, R., Chionchio, G., Di Lellis, A., Marcucci, M. F., Pallocchia, G., Korth, A., Daly, P. W., Graeve, B., Rosenbauer, H., Vasyliunas, V., McCarthy, M., Wilber, M., Eliasson, L., Lundin, R., Olsen, S., Shelley, E. G., Fuselier, S., Ghielmetti, A. G., Lennartsson, W., Escoubet, C. P., Balsiger, H., Friedel, R., Cao, J.-B., Kovrazhkin, R. A., Papamastorakis, I., Pellat, R., Scudder, J., and Sonnerup, B.: First multispacecraft ion measurements in and near the Earth's magnetosphere with the identical Cluster ion spectrometry (CIS) experiment, Ann. Geophys., 19, 1303-1354, doi:10.5194/angeo19-1303-2001, 2001.

Rosenbauer, H., Gruenwaldt, H., Montgomery, M. D., Paschmann, G., and Sckopke, N.: Heos 2 plasma observations in the distant polar magnetosphere - The plasma mantle, J. Geophys. Res.Space, 80, 2723-2737, doi:10.1029/JA080i019p02723, 1975.

Savin, S. P., Zelenyi, L. M., Romanov, S. A., Klimov, S. I., Skalsky, A. A., Galeev, A. A., Smirnov, V. N., Nozdrachev, M. N., Yermolaev, Y. I., Avanov, L. A., Amata, E., Blecki, J., Buechner, 
J., Nikutowski, B., Dubinin, É. M., Nemecek, Z., Safrankova, J., Pedersen, A., Rauch, J. L., Rustenbach, J., Sauvaud, J. A., Song, P., and Stasiewicz, K.: Turbulent Boundary Layer at the Border of Geomagnetic Trap, Soviet Journal of Experimental and Theoretical Physics Letters, 74, 547-551, doi:10.1134/1.1450288, 2001.

Smith, M. F. and Lockwood, M.: Earth's magnetospheric cusps, Rev. Geophys., 34, 233-260, doi:10.1029/96RG00893, 1996. vom Stein, R., Glassmeier, K.-H., and Dunlop, M.: A Configuration Parameter for the Cluster Satellites, Tech. Rep. Tech. Rep. 2/1992, Institut für Geophysik und Meteologie der Technischen Universität Braunschweig, 1992. 